\title{
Index to Volume 134
}

Compiled by William Halliday

Acorn, J., 205

Activity,

Daily, 353

Seasonal, 248

Aegolius

acadicus, 125

funereus, 125

Alberta,

Clear Hills, 125

Edmonton, 205

Rocky Mountains, 241

Alces americanus, 361

Archer, S.K., G. Dennison, L. Tryon, S. Byers, A. Dunham. Invertebrate settlement and diversity on a glass sponge reef, 1-15

Armstrong, T., 213

Asymmetry,

Fluctuating, 136

Skeletal, 136

Autumn, 248

Baryluk, S., 144

Bat, Little Brown, 248

Bayne, E.M., 125, 136

Beaver, American, 342

Bees, Bumble, 16

Beetle, Burying, 217

Behaviour, 132

Aggregation, 175

Epimeletic, 316

Hunting, 42, 205

Nesting, 329

Vocal, 117

Béland, P., 21

Beluga, 21

Bergerud, A.T., B.E. McLaren, W. Dalton, L. Camps, H. Butler, R.S. Ferguson. Changes among Caribou (Rangifer tarandus) in Slate Islands Provincial Park following successive arrivals of Gray Wolves (Canis lupus), 342-352

Beringia, 231

Bill, H., 42

Bioacoustics, 117

Biodiversity, 165

Bird, 117, 125, 136, 152, 205, 217, 321, 329

Bison, 161

Bison bison, 161

Bivalve, 179

Bombus, 16
Boar, Eurasian Wild, 132

Boreal, 125, 329

Boxes, Nest, 125

Bradley, D.W., 152

British Columbia, 1

Central, 265

Lower Mainland, 60, 210

North-central, 248, 361

North Thompson River, 252

Prince George, 152

Salish Sea, 316

Brochez, C.B., R.V. Rea, S.M. Crowley, D.P. Hodder. Year-round patterns of mineral lick use by Moose (Alces americanus), deer, and Elk (Cervus canadensis) in north-central British Columbia, 361-374

Brodo, I., 113

Brome, Smooth, 156

Bromus inermis, 156

Brook, R.K., M.J. Clarke. Interactions between Elk (Cervus canadensis) and invasive Feral Swine (Sus scrofa) on the Canadian Prairies, 132-135

Browne, C.L., S.A. Sullivan, D.F. McAlpine. Freshwater turtle by-catch from angling in New Brunswick, Canada, 222-230

Brunton, D.F., M.A. Krichbaum, R.S. Krichbaum, P.C. Sokoloff. Distribution and status of Howell's Quillwort (Isoetes howellii, Isoetaceae) in Canada and its relation to Bolander's Quillwort (Isoetes bolanderi), 252-264

Brunton, D.F., P.M. Catling, B. Kostiuk. Tribute to "The Snake Man", Francis Russell Cook, Ph.D., C.M. (1935-2020), 71-84

Bullsnake, 175

Burden, Mercury, 136

Burrow, 329, 353

Butler, H., 342

By-catch,

Angling, 222

Fishing, 222

Byers, S., 1

Camera, Trail, 132

Cameron, A.D.S., 16

Camps, L., 342

Canada, Maritime, 375

Canis

latrans, 36

lupus, 36, 342 
Cannings, R.A., T. Goward, W.F. Merilees. Tribute to

R. Yorke Edwards, 1924-2011, 270-294

Card, J., 205

Carex

duriuscula, 231

filifolia, 231

lupuliformis, 171

Caribou, 342

Northern Mountain, 265

Caskenette, A.L., 64

Castor canadensis, 342

Catharus fuscescens, 321

Catling, P.M., 71

Catling, P.M. Carolyn Callaghan-stop stepping down!, 404

Catling, P.M., B. Kostiuk, T. Armstrong. New records of grasshoppers (Orthoptera) from the Northwest Territories, 213-216

Catling, P.M., B. Kostiuk. Disjunctions of Needleleaved Sedge (Carex duriuscula) and Threadleaved Sedge (Carex filifolia) in the Husky Lakes area of Northwest Territories-first records for the Canadian true Arctic and possible Pleistocene relicts, 231-240

Certhia americana, 117

Cervus canadensis, 132, 241, 361

Cetacean, 21

Change, Climate, 52 Ecosystem, 21

Charles, C., 64

Chelydra serpentina, 56, 222

Chorus, Dawn, 117

Chrysemys picta, 56, 222 picta picta, 222

Cicadellidae, 156

Cipriani, J., 113

Circadian, 353

Clam, Asian, 179 Fingernail, 56

Clarke, M.J., 132

Columba livia, 205

Competition, Apparent, 265

Concealment, 152

Connell, C.B., 375

Consiglio, J.A., M.J. Oldham. An update to the distribution of the Endangered False Hop Sedge (Carex lupuliformis Sartwell ex Dewey; Cyperaceae) in Ontario, 171-174

Contact, Indirect, 132

Contents, Stomach, 64
Corbicula fluminea, 179

Cougar, 265

Court, G., 205

Cover, Foliage, 156

Coyote, 36

Crayfish, Southern White River, 375

Creeper, Brown, 117

Crithidia expoeki, 16

Crowley, S.M., 361

Curlew, Long-billed, 152

Cyperaceae, 171, 231

Dalley, K.L., 307

Dalton, W., 342

Deer, Mule, 361

White-tailed, 361

DeGroot, L., 265

Delaney, D., 205

Delphinapterus leucas, 21

DeMarco, K.V.B., P.R. Martin. A case of a Pustulated Carrion Beetle (Nicrophorus pustulatus, Coleoptera: Silphidae) burying live Tree Swallow (Tachycineta bicolor, Passeriformes: Hirundinidae) nestlings under the nest, 217-221

Dennison, G., 1

Deichsel, G., 60

Diet, 21, 64

Diplocolenus configuratus, 156

Distribution, 36, 52, 60, 144, 213, 307

Canadian, 16

Disturbance, Anthropogenic, 52

Diversity, 1 Insect, 156

Domahidi, Z., S.E. Nielsen, E.M. Bayne, J.R. Spence. Boreal Owl (Aegolius funereus) and Northern Saw-whet Owl (Aegolius acadicus) breeding records in managed boreal forests, 125-131

Doratura stylata, 156

Doridoxa ingolfiana, 165

Doridoxidae, 165

Draft Minutes of the $141^{\text {st }}$ Annual Business Meeting (ABM) of the Ottawa Field-Naturalists' Club, 14 January 2020, 103-112

Dreissena polymorpha, 179

rostriformis bugensis, 179

Drummond, R., 161

Dunham, A., 1

Dynamics, Predator-prey, 265

Ecology, 213

Evolutionary, 217 
Foraging, 21

Nesting, 329

Winter, 161

Editors' Report for Volume 133 (2019), 201-204

Eider, Common, 136

Elk, 132, 241, 361

Elliptio, Eastern, 56

Elliptio complanata, 56

Enders, E.C., T. Nagalingam, A.L. Caskenette, T.A.

Rudolfsen, C. Charles, D.A. Watkinson. Diet of a rare Canadian fish species, Carmine Shiner

(Notropis percobromus) in the Birch River,

Manitoba, Canada, 64-70

Equus ferus caballus, 265

Estuary, St. Lawrence, 21

Expansion, Range, 52, 60, 144, 171, 179, 210, 213

Extension, Range, 52, 60, 144, 171, 179, 210, 213, 307

\section{Falco}

mexicanus, 205

rusticolus, 205

Falcon,

Prairie, 205

Winter, 205

Farrell, R., G. Hanke, D. Veljacic. First verified sighting of a Western Fence Lizard (Sceloporus occidentalis) in British Columbia, Canada, 210-212

Ferguson, R.S., 342

Fidelity, Nest Site, 329

Fish, 64, 307

Fishing, 42, 222

Foliage, 156

Foote, J.R., 117

Foraging, 42

Forbes, G.J., D.F. McAlpine. An assessment of historical records of Wolverine (Gulo gulo) from New Brunswick, 147-151

Forest, Boreal, 125

Fox, Red, 144, 342

Freemartinism, 241

Fuirst, M., 42

Fundulus

diaphanus, 307

heteroclitus, 307

Gilchrist, H.G., 136

Glyptemys insculpta, 56, 222

Gordon, D.C., 144

Gordon, Jr., D., 144

Goward, T., 270

Grass, 156

Grasshopper(s), 213

Two-striped, 213
Grassland, Fescue, 156

Grief, Animal, 316

Gulo gulo, 147

Gyrfalcon, 205

Habitat, Nesting, 321, 329

Hamel, J.-F., 165

Hanke, G.F., G. Deichsel. First record of an Italian Wall Lizard (Podarcis siculus) in British Columbia, Canada, 60-63

Hanke, G.F., 210

Hanrahan, C., 113

Haplotypes, Coyote-wolf, 36

Hare, Snowshoe, 342

Harms, N.J., 161

Harpley, P., 321

Hawey, C., P. Harpley, R. Milne. Habitat use by Veery (Catharus fuscescens) in southern Ontario, 321-328

Hazelton, P.D., 56

Hebblewhite, M., 241

Hedstrom, H., 205

Hemiptera, 156

Hermaphroditism, 241

Herpetology, 175

Hibernation, 248

Hodder, D.P., 361

Hollyoake, A.L., 152

Horses, Feral, 265

Hunting, 42, 205

Huxter, C.E., 248

Hybridization, Coyote-wolf, 36

Hymenoptera, 52

Ice, Sea, 144

Identification, 307

Illinois, 36

iNaturalist, 210

Insectivore, Aerial, 329

Intensity, Parasite, 136

Interactions, 132

Invertebrate,

Freshwater, 375

Marine, 1, 165

Terrestrial, 16, 156, 213, 217

Isoetes

bolanderi, 252

howellii, 252

Johnson, N.B., J.L. Van Parys, C.M. Somers, R.G. Poulin. A report of unusual aggregation behaviour in Bullsnakes (Pituophis catenifer sayi) near a nest site in Saskatchewan, 175-178 
Johnson, S.L., 56

Jones, M.T., L.L. Willey, D.T. Yorks, P.D. Hazelton, S.L. Johnson. Passive transport of Eastern Elliptio (Elliptio complanata) by freshwater turtles in New England, 56-59

Jung, T.S., M.J. Suitor, S. Baryluk, J. Nuyaviak, D.C. Gordon, D. Gordon, Jr., E. Pokiak. Red Fox (Vulpes vulpes) scavenging on the spring sea ice: potential implications for Arctic food webs, 144-146

Jung, T.S., R. Drummond, N.J. Harms. Bison (Bison bison) activity fragments subnivean tunnels of small mammals, 161-164

Killifish, Banded, 307

Kostiuk, B., 71, 213, 231

Krichbaum, M.A., 252

Krichbaum, R.S., 252

Lair, S., 21

Lake(s), Great, 36, 179

Simcoe, 321

Larson, S., 316

Lepage, D. Draft Minutes of the $141^{\text {st }}$ Annual Business Meeting (ABM) of the Ottawa Field-Naturalists' Club, 14 January 2020, 103-112

Lepitzki, D.A.W. In Memoriam: Ronald E. Bedford (26 June 1930-3 November 2020), 405

Lepitzki, D.A.W. In Memoriam: Donald A. Smith (29 August 1930-13 November 2020), 405

Lepitzki, D.A.W., A.E. Martin. Editors' Report for Volume 133 (2019), 201-204

Lepus americanus, 342

Lesage, V., S. Lair, S. Turgeon, P. Béland. Diet of St. Lawrence Estuary Beluga (Delphinapterus leucas) in a changing ecosystem, 21-35

Lewis, J.H. Sphex ichneumoneus and Sphex pensylvanicus (Hymenoptera: Sphecidae) in Atlantic Canada: evidence of recent range expansion into the region, 52-55

Lick, Mineral, 361

Lilliput, 179

Lizard, Italian Wall, 60

Western Fence, 210

Locust, Heron Short-winged, 213

Logging, Partial, 125

Lupulinae, 171

Lynds, M., J. Card, H. Hedstrom, D. Delaney, G. Court, J. Acorn. Large winter falcons and their Rock Pigeon (Columba livia) prey at an urban grain terminal in Edmonton, Alberta: an update, 205-209
Mammal,

Arboreal, 248

Terrestrial, 36, 42, 132, 144, 147, 161, 241, 265, $342,353,361$

Marine, 21, 316

Small, 161

Management, Forest, 125

Manitoba,

Southeastern, 64

Southern, 156

Mapleleaf, 179

Marini, K.L.D., S. Nadon, J.R. Foote. Dawn singing in Brown Creeper (Certhia americana), 117-124

Marmot, 353

Marmota monax, 353

Martin, A.E., 201

Martin, A.E. Dan Brunton steps down from the Ottawa Field Naturalists' Club Publications Committee after many years of service, 405

Martin, H., 241

Martin, P.R., 217

McAlpine, D.F., 147, 222

McAlpine, D.F. A tribute to Paul-Michael Brunelle, odonatologist, 1952-2020, 379-386

McAlpine, D.F., C.B. Connell, P.D. Seymour. Introduction of Southern White River Crayfish (Procambarus zonangulus) to New Brunswick, 375378

McGonigle, T.P., 156

McLachlan Hamilton, K., 113

McLaren, B.E., 342

McNichols-O'Rourke, K.A., 179

Melanoplus

bivittatus, 213

huroni, 213

Memoriam, 405

Mercier, A., 165

Mercury, 136

Merilees, W.J., 270

Merrill, E.H., 241

Microtus 161 pennsylvanicus, 161 oeconomus, 161

Milne, R., 321

Minnesota, 36

Minutes of the $141^{\text {st }}$ Annual Business Meeting (ABM) of the Ottawa Field-Naturalists' Club, 14 January 2020, 103-112

Monitoring, Passive Acoustic, 117

Trail Camera, 132

Mollusca, 165

Moose, 361 
Morris, T.J., M.N. Sheldon, K.A. McNichols-O'Rourke. First records of two freshwater mussel Species at Risk, Mapleleaf (Quadrula quadrula) and Lilliput (Toxolasma parvum), in the Canard River, Ontario, with implications for freshwater mussel recovery in the Detroit River, 179-188

Mossop, M.D., 329

Mummichog, 307

Mussels,

Freshwater, 56, 179

Quagga, 179

Zebra, 179

Myotis, Little Brown, 248

Myotis lucifugus, 248

Nadon, S., 117

Nagalingam, T., 64

National Park, Riding Mountain, 156

Nesting, 125, 152, 175, 321, 329

New Brunswick, 147, 375

Southern, 52, 222

New England, 56

Newfoundland and Labrador, 165, 307

Insular, 307

Nicrophorus pustulatus, 217

Nielsen, S.E., 125

Normandeau, J., H. Martin, E.H. Merrill, M. Hebblewhite. Potential case of pseudo-hermaphroditism in Elk (Cervus canadensis) in Alberta, Canada, 241-247

Northey, A., 316

Northwest Territories, 213, 231

Beaufort Sea, 144

Notropis percobromus, 64

Nudibranchs, Deep-sea, 165

Numenius americanus, 152

Nunavut, Cape Dorset, 136

Nuyaviak, J., 144

Occupancy, Colony, 329

Ocean,

North Atlantic, 165

Pacific, 1

Odocoileus

hemionus, 361

virginianus, 361

Oldham, M.J., 171

Ontario,

Eastern, 217

Northern, 117, 342

Southern, 42, 171, 179, 321, 353

Orca, 316

Orcinus orca, 316
Orthoptera, 213

Osborne, D.R., 307

Ovenden, L., 113

Owl,

Boreal, 125

Northern Saw-whet, 125

Palmier, K.M., A.D. Tripodi, A.D.C. Cameron, J.P. Strange, C.S. Sheffield. First record of Crithidia expoeki (Trypanosomatida: Trypanosomatidae) from native Canadian bumble bees (Hymenoptera: Apidae: Bombus), 16-20

Panopi, J.G., 136

Parasite, 136

Gastrointestinal, 136

Park, Slate Islands Provincial, 342

Pathogen, Protozoan, 16

Pattern, Activity, 353

Penney, H.D., J.-F. Hamel, A. Mercier. Range extension of two deep-sea nudibranchs, Tritonia newfoundlandica and Doridoxa ingolfiana (Mollusca: Gastropoda: Heterobranchia), in eastern Canada, $165-170$

Phenology, Nesting, 329

Phytogeography, 231, 252

Pig, Feral, 132

Pigeon, Rock, 205

Pituophis catenifer sayi, 175

Plant, 156, 171, 231, 252

Podarcis siculus, 60

Pokiak, E., 144

Pollinator, 16

Porifera, 1

Poulin, R.G., 175

Predation, 42, 205, 265

Preference, Colour, 205

Prey, 205, 265

Procambarus zonangulus, 375

Protozoan, 16

Provencher, J.F., 136

Pseudo-hermaphroditism, 241

Puma concolor, 265

Quadrula quadrula, 179

Quebec, Southern, 21

Quillwort,

Bolander's, 252

Howell's, 252

Range, Geographic, 52, 307

Rangifer tarandus 342

caribou, 265 
Rea, R.V., 361

Rea, R.V., C.E. Huxter. Late-autumn record of Little Brown Myotis (Myotis lucifugus) in north-central British Columbia, 248-251

Record,

Breeding, 125

First, 60, 210, 213

Historical, 147

Recording, Autonomous, 117

Recovery, 179

Reef, Glass Sponge, 1

Region, Boreal, 329

Relict, 231

Reptile, 60, 175, 210, 222

Reuse, Burrow, 329

Riparia riparia, 329

River,

Birch, 64

Canard, 179

Detroit, 179

Thompson, 252

Rosenkranz, V.E., T.P. McGonigle. Reductions in foliar Hemiptera in portions of a fescue grassland invaded by Smooth Brome (Bromus inermis), 156160

Rudolfsen, T.A., 64

Sargent, P.S., K.L. Dalley, D.R. Osborne. Banded Killifish (Fundulus diaphanus) and Mummichog (Fundulus heteroclitus) distributions in insular Newfoundland waters: implications for a Species at Risk, 307-315

Saskatchewan, 16

Central, 132

Southern, 175

Scavenging, 144

Sceloporus occidentalis, 210

Sciurus carolinensis, 42

Scobie, C.A., J.G. Panopi, E.M. Bayne, J.R. Provencher, H.G. Gilchrist. No relationship between gastrointestinal parasite intensities or mercury burdens on fluctuating asymmetry in Common Eider (Somateria mollissima) mandibles, 136-143

Sea,

Beaufort, 144

Salish, 316

Sedge, False Hop, 171

Settlement, Juvenile, 1

Seymour, P.D., 375

Shedd, T., A. Northey, S. Larson. Epimeletic behaviour in a Southern Resident Killer Whale (Orcinus orca), 316-320

Sheffield, C.S., 16
Sheldon, M.N., 179

Shiner, Carmine, 64

Shores, C.R., 265

Sighting, First, 210

Sinclair, P.H., M.D. Mossop, S.A. Stotyn. Nesting ecology and reuse of nest burrows by Bank Swallow (Riparia riparia) in southern Yukon, 329-341

Singing, Dawn, 117

Size, Clutch, 125

Slider, Red-eared, 222

Slug, Sea, 165

Snake, 175

Snow, 161

Sokoloff, P.C., 252

Somateria mollissima, 136

Somers, C.M., 175

Sorenson, G.H., A.L. Hollyoake, D.W. Bradley. First confirmed nest of Long-billed Curlew (Numenius americanus) in roadside brush stubble, 152-155

Species,

Aquatic Invasive, 375

Introduced, 60, 132, 375

Invasive, 132, 156, 179, 375

Species at Risk, 21, 64, 171, 175, 179, 252, 307, 329

Spence, J.R., 125

Sphaerium sp., 56

Sphecidae, 52

Sphex

ichneumoneus, 52

pensylvanicus, 52

Sponge, Glass, 1

Squirrel, Eastern Gray, 42

Steen, R. Diel activity patterns of urban Woodchucks (Marmota monax) revealed by camera traps at burrows in southwestern Ontario, Canada, 353-360

Stotyn, S.A., 329

Strange, J.P., 16

Structure, Community, 1

Subnivean, 161

Success, Nestling, 125

Suitor, M.J., 144

Sullivan, S.A., 222

Survey,

Aerial, 144

Camera Trap, 353, 361

Radio Telemetry, 175, 265

Transect, 231, 321

Trawl, 165

Sus scrofa, 132

Sutton, A.O., M. Fuirst, K. Bill. Into the drink: observation of a novel hunting technique employed 
by an Eastern Gray Squirrel (Sciurus carolinensis), 42-44

Swallow,

Bank, 329

Tree, 217

Swimming, 42

Swine, Feral, 132

Sympatry, Coyote-wolf, 36

Tachycineta bicolor, 217

Taxonomy, 165

Temperature, 205, 248

Terminal, Grain, 205

The Ottawa Field-Naturalists' Club Awards for 2019, presented February 2020, 113-115

Thermoregulation, 205

Thiel, R.P. Clarifying late Holocene Coyote (Canis latrans)-Gray Wolf (Canis lupus) sympatry in the western Great Lake states, 36-41

Threats, 222

Toxolasma parvum, 179

Trachemys scripta, 222

Transport, 56

Trap, Camera, 353, 361

Tribute, 71, 270, 379

Tribute to "The Snake Man", Francis Russell Cook, Ph.D., C.M. (1935-2020), 71-84

Tribute to R. Yorke Edwards, 1924-2011, 270-294

Tribute to Paul-Michael Brunelle, odonatologist, 19522020, 379-386

Tripodi, A.D., 16

Tritonia newfoundlandica, 165

Tritoniidae, 165

Tundra-steppe, 231

Turgeon, S., 21

Turtle,

Eastern Painted, 56, 222

Freshwater, 56, 222

Red-eared Slider, 222
Snapping, 56, 222

Wood, 56, 222

Tryon, L., 1

Ungulate, 132, 241, 342, 361

Unionidae, 56, 179

Urban, 353

Use, Habitat, 321

Van Parys, J.L., 175

Veery, 321

Veljacic, D., 210

Voles, 161

Meadow, 161

Tundra, 161

Vulpes vulpes, 144, 342

Warming, Climate, 52

Washington, Salish Sea, 316

Wasp, 52

Thread-waisted, 52

Watkinson, D.A., 64

Whale, Southern Resident Killer, 316

White, S.C., C.R. Shores, L. DeGroot. Cougar (Puma concolor) predation on Northern Mountain Caribou (Rangifer tarandus caribou) in central British Columbia, 265-269

Willey, L.L., 56

Wisconsin, 36

Wolf, Gray, 36, 342

Wolverine, 147

Woodchuck, 353

Yorks, D.T., 56

Yukon, 161, 329

Zurbrigg, E., I. Brodo, J. Cipriani, C. Hanrahan, L. Ovenden, K. McLachlan Hamilton. The Ottawa Field-Naturalists' Club Awards for 2019, presented February 2020, 113-115 


\section{Index to Book Review}

\section{Biology}

Cottam, B. "The Inside Out of Flies" by Erica McAlister, 2020, 298

Cottam, B. "Chasing Nature: An Ecologist's Lifetime of Adventures and Observations" by Robert E. Wrigley. Illustrated by Rob Gillespie, 2020, 300 301

Cray, H. "The Call of Carnivores: Travels of a Field Biologist" by Hans Kruuk, 2019, 299

Halliday, W.D. "Whales of the Southern Ocean: Biology, Whaling and Perspectives of Population Recovery. Advances in Polar Ecology Volume 5" by Yuri Mikhalev, 2020, 189

Way, J. "The Cougar Conundrum: Sharing the World with a Successful Predator" by Mark Elbroch, 2020, 295-297

\section{Botany}

Brunton, D.F. "Flora of Florida, Volume VII: Dicotyledons, Orobanchaceae through Asteraceae" by Richard P. Wunderlin, Bruce F. Hansen, and Alan R. Franck, 2020, 85-86

Clark, Jr., H.O. "Darwin's Most Wonderful Plants: A Tour of His Botanical Legacy" by Ken Thompson, 2019, 190-191

\section{Conservation and Wildlife}

Forbes, G.J. "The Wildlife Techniques Manual (Eighth Edition). Volume 1 - Research. Volume 2 - Management" by Nova J. Silvy, 2020, 387-388

\section{Entomology}

Burke, T. "Raising Butterflies in the Garden" by Brenda Dziedzic, 2019, 192-193

\section{Herpetology}

Halliday, W.D. "Secrets of Snakes: The Science Beyond the Myths. W.L. Moody, Jr., Natural History Series" by David A. Steen, 2019, 193

\section{Ornithology}

Alvo, R. "Waterfowl of Eastern North America. Second Edition" by Chris G. Earley, 2020, 389

Gaston, T. "Birds in Winter: Surviving the Most Challenging Season" by Roger F. Pasquier. Illustrated by Margaret La Farge, 2019, 87

Gaston, T. "Oceanic Birds of the World: A Photo Guide" by Steve N.G. Howell and Kirk Zufelt, 2019, 194

\section{Other}

Charles, G. "A Sand County Almanac and Sketches Here and There" by Aldo Leopold. Introduction by Barbara Kingsolver, 2020, 195-196

Cottam, B. Books in Brief, 96-97, 398

Cottam, B. New Titles, 98-100, 197-199, 302-305, 399-402

Cray, H. "The Tangled Tree: A Radical New History of Life" by David Quammen, 2018, 90

Foster, R.F. "Levelling the Lake: Transboundary Resource Management in the Lake of the Woods Watershed" by Jamie Benidickson, 2019, 91-92

Halliday, W.D. "Brave New Arctic: The Untold Story of the Melting North" by Mark C. Serreze, 2018, 396

Tegler, B. "The Mosquito: A Human History of Our Deadliest Predator" by Timothy C. Winegard, 2019, 92-93

Tegler, B. "Horizon" by Barry Lopez, 2019, 396-397

Way, J. "Re-Bisoning the West: Restoring an American Icon to the Landscape" by Kurt Repanshek, 2019, 94-95

\section{Zoology}

Halliday, W.D. "Marine Mammals: Adaptations for an Aquatic Life" by Randall W. Davis, 2019, 88

Halliday, W.D. "The Voices of Marine Mammals: William E. Schevill and William A. Watkins: Pioneers in Bioacoustics" edited by Christina Connett Brophy, 2019, 89

Halliday, W.D., N. Diogou, A.F. Heimrich, M.J. Martin. "The Bowhead Whale Balaena mysticetus: Biology and Human Interactions" edited by J.C. George and J.G.M. Thewissen, 2020, 390-391

Way, J. "The Reign of Wolf 21: The Saga of Yellowstone's Legendary Druid Pack" by Rick McIntyre, 2020, 392-393

Way, J. "Wolf Island: Discovering the Secrets of a Mythic Animal" by L. David Mech and Greg Breining, 2020, 394-395 